\title{
Hilbert 变换相关的分布空间及其应用
}

\author{
杨力华
}

中山大学数学与计算科学学院, 广州 510275

E-mail: mcsylh@mail.sysu.edu.cn

收稿日期: 2007-04-17; 接受日期: 2007-09-16

国家自然科学基金 (批准号: 60475042,10631080) 资助项目

摘要本文构造了一个新的分布空间 $\mathscr{D}_{H}^{\prime}$, 并且把经典的 Hilbert 变换的定义推广到这个 空间上. 证明了推广的 Hilbert 变换是把空间 $\mathscr{D}_{H}^{\prime}$ 映射到自身的同胚映射, 且 $\mathscr{D}_{H}^{\prime}$ 是满足该 条件的 $\mathscr{D}^{\prime}$ 的最大子空间. 进一步, 证明了 $L^{p}$ 空间中经典的 Hilbert 变换和周期函数空间中 周期 Hilbert 变换都是这一推广 Hilbert 变换的特款. 对空间 $\mathscr{D}_{H}$ 的性质进行了刻画，并给 出了空间 $\mathscr{D}_{H}^{\prime}$ 中的一类非常有用的非线性相位信号. 最后给出了该推广 Hilbert 变换的两个 简单的应用。

关键词 Hilbert 变换 分布 时频分析

$\mathrm{MSC}(2000)$ 主题分类 $44 \mathrm{~A} 15,46 \mathrm{~F} 12$

\section{1 引言}

在物理、数学以及工程等诸多领域中, Fourier 变换和 Hilbert 变换是两个非常重要的变 换. Fourier 变换在经典信号处理的频谱分析中处于无可置疑的主导地位 (文献 [1, 第 1 章]). 同样地, Hilbert 变换由于能够提取信号的瞬时频率信息, 为非线性信号分析提供了坚实的理 论基础. 对一个实信号做 Hilbert 变换, 然后以此为虚部加到原信号上, 就构成了原信号的解 析信号. 利用解析信号的复数表示便可以定义信号的瞬时振幅和瞬时频率, 这是一种迄今为 止广为接受的定义瞬时频率的方法 ${ }^{[2]}$. 实际应用中常见的信号主要有能量有限信号 (即数学 中的 $L^{2}$ 函数)、周期信号以及 Dirac 脉冲函数 (即广义函数, 亦称为分布) 等. 因此当对信号 进行严格的数学分析时, 我们希望能建立一个包含上述信号的函数空间, 并使得 Fourier 变换 或 Hilbert 变换在这个空间上是封闭运算. 众所周知, 做 Fourier 变换分析的理想空间是缓增 分布空间, 它包含上述各类信号且使得 Fourier 变换在该空间上是同胚的. 然而, 对于 Hilbert 变换, 我们还没有这样的一个类似的空间. 随着信息科学的发展, Hilbert 变换在非平稳信号 处理中发挥着越来越重要的作用. 最近由 N. E. Huang 等人所提出的 Hilbert-Huang 变换, 就应用 Hilbert 变换产生一种基于所谓经验模式分解的 Hilbert 谱 ${ }^{[3]}$. 该方法激起了人们对 
Hilbert 变换以及相关问题, 比如 Bedrosian 等式, 的重新认识和研究 ${ }^{[4-7]}$.

经典 Hilbert 变换的定义如下:

$$
H f(t):=\frac{1}{\pi} \text { p.v. } \int_{\mathbb{R}} \frac{f(t-\tau)}{\tau} d \tau,
$$

其中 p.v. 表示 Cauchy 主值. 对任意 $1<p<\infty, H$ 是空间 $L^{p}(\mathbb{R})$ 到其自身的连续线性算子, 并且满足下面的基本性质 ${ }^{[8,9]}$ : (i) $H^{-1}=-H: L^{2}(\mathbb{R}) \rightarrow L^{2}(\mathbb{R})$; (ii) $\|H f\|_{2}=\|f\|_{2}(\forall f \in$ $\left.L^{2}(\mathbb{R})\right)$; (iii) $(H f)^{\wedge}(\omega)=-i(\operatorname{sgn} \omega) \hat{f}(\omega)$ 对几乎处处 $\omega \in \mathbb{R}$ 和任意 $f \in L^{2}(\mathbb{R})$ 成立, 其中 $\hat{f}$ 是 $f$ 的 Fourier 变换, 当 $f \in L^{1}(\mathbb{R})$ 时, 其定义为

$$
\hat{f}(\omega):=\int_{\mathbb{R}} f(x) \mathrm{e}^{-i \omega x} d x .
$$

当 $f \in L^{2}(\mathbb{R})$ 时, 其 Fourier 变换通过 $L^{2}(\mathbb{R}) \cap L^{1}(\mathbb{R})$ 在 $L^{2}(\mathbb{R})$ 中的稠密性来定义 ${ }^{[10]}$. 类似 地, 对任意周期为 $T>0$ 的周期函数 $f$, 其周期 Hilbert 变换的定义如下:

$$
\tilde{H} f(x):=\frac{1}{\pi} \text { p.v. } \int_{-T / 2}^{T / 2} \frac{f(t-\tau)}{2 \tan \frac{\tau}{2}} d \tau .
$$

我们把式 (1.1) 和 (1.2) 中定义的 $H$ 和 $\tilde{H}$ 统称为 Hilbert 变换. 一个有趣而自然的问题是 $H$ 和 $\tilde{H}$ 有怎样的关系? 对 $f \in L^{2}(\mathbb{R})$, 若 $s(t)=f(t)+\cos t$, 则 $s$ 的 Hilbert 变换是什么, 如何求 它的瞬时频率?

尚未见到关于上述问题研究的报道和文献. 然而, 将 Hilbert 变换推广到广义函数空间却 有许多研究, 例如文献 [11-16]. 这些推广的主要方法是利用分布的解析表示把 Hilbert 变换 推广到已有的某个分布空间 ${ }^{[11-13]}$. 在这些工作中, 尤其要提到 Orton 的工作, 她在文献 [13] 中利用解析表示将经典的 Hilbert 变换推广到了 Schwartz 分布空间 $\mathscr{D}^{\prime}$ 中, 而且她所定义的 Hilbert 变换在模整函数的意义下是唯一的, 即照此定义, $f \in \mathscr{D}^{\prime}$ 的 Hilbert 变换事实上是一 个等价类. 在文献 [14] 中, Pandey 通过在空间 $H(\mathscr{D})$ 中引入拓扑, 再直接利用共轭算子的方 法, 将 Hilbert 变换推广到空间 $\mathscr{D}^{\prime}$ 上. 在这种定义下, 任意 $f \in \mathscr{D}^{\prime}$ 的 Hilbert 变换 $H f$ 都属 于所谓超分布空间 $H^{\prime}(\mathscr{D})$. 由于对任意非零的 $\phi \in \mathscr{D}$, 总有 $H \phi \notin \mathscr{D}$, 所以 $H^{\prime}(\mathscr{D})$ 不是 $\mathscr{D}^{\prime}$ 的子空间. 这样, Pandey 所定义的 $H f$ 不是 Schwartz 意义下的广义函数. 类似的情形其实 对 Fourier 变换也存在, 因为对任意非零的 $\phi \in \mathscr{D}$, 其 Fourier 变换也不属于 $\mathscr{D}$. 所以, 为了 把 Fourier 变换延拓到适当的分布空间, 人们考虑了速降函数类 $\mathscr{S}$. 我们知道, $\mathscr{D} \subset \mathscr{S}$ (嵌入 ' $\square$ ' 的准确意义参见文献 [17]), 且 Fourier 变换在空间 $\mathscr{S}$ 上是同胚的, 因而 $\mathscr{S}$ 的对偶空间 满足 $\mathscr{S}^{\prime} \hookrightarrow \mathscr{D}^{\prime}$. 于是, 利用共轭算子方法, 人们把 Fourier 变换成功地推广到了空间 $\mathscr{S}^{\prime}$ 上. 沿 用这个想法, 本文将构造一个新的分布空间, 并把经典 Hilbert 变换推广到该分布空间, 使得 该推广的 Hilbert 变换是这个空间上的一个同胚映射. 而且, 我们还将证明分别通过式 (1.1) 和 (1.2) 定义的 Hilbert 变换均为该推广 Hilbert 变换的特款.

为表述清晰, 对下文中几个常用记号说明如下: $\mathbb{N}$ 表示所有自然数的集合, $\mathbb{Z}_{+}$表示所 有非负整数的集合, $\mathbb{R}$ 表示实数集. 对 Lebesgue 测度集 $E \subset \mathbb{R}$ 和 $1 \leqslant p \leqslant \infty, L^{p}(E)$ 表 示 $p$ 次 Lebesgue 可积函数空间, $L^{p}(E)$ 空间上赋予了周知的 $L^{p}(E)$ 范数, $L_{\mathrm{loc}}(\mathbb{R})$ 表示定义 在 $\mathbb{R}$ 上的局部可积函数所构成的空间, $C^{k}(\mathbb{R})\left(k \in \mathbb{Z}_{+}\right)$是 $\mathbb{R}$ 上的 $k$ 次连续可微函数空间, $C(\mathbb{R}):=C^{0}(\mathbb{R}), C^{\infty}(\mathbb{R}):=\bigcap_{k \in \mathbb{N}} C^{k}(\mathbb{R}), \mathscr{D}$ 表示所有紧支集 $C^{\infty}(\mathbb{R})$ 函数所构成的试验函数 空间, 空间 $\mathscr{D}$ 配备周知的拓扑后, 其对偶空间 $\mathscr{D}^{\prime}$ 形成 Schwartz 分布空间 ${ }^{[17]}$. 


\section{2 空间 $\mathscr{D}_{H}$}

将经典 Hilbert 变换推广到某个分布空间 $\mathscr{X}^{\prime}$ 的典型方法是共轭算子方法, 其中 $\mathscr{X}$ 是 某个函数空间, $\mathscr{X}^{\prime}$ 是其对偶空间. 我们把要构造的函数空间记为 $\mathscr{D}_{H}$, 假设它满足 $\mathscr{D} \subset \mathscr{D}_{H}$ 和 $H\left(\mathscr{D}_{H}\right)=\mathscr{D}_{H}$, 则 $\mathscr{D}, H(\mathscr{D}) \subset \mathscr{D}_{H}$, 因此 $\mathscr{D}+H(\mathscr{D}) \subset \mathscr{D}_{H}$. 一般情形下, 空间 $\mathscr{D}_{H}$ 越小, 其 对偶空间 $\mathscr{D}_{H}^{\prime}$ 就越大. 本文中定义

$$
\mathscr{D}_{H}:=\mathscr{D}+H(\mathscr{D}) .
$$

下面将证明 $\mathscr{D}_{H}$ 正是我们所需构造的空间.

以下, 我们用 $C(A)$ 表示只依赖于 $A$ 的一个非负常数, 但在不同的地方可能取不同的值. 这里的 $A$ 可以是某些已知的数、函数、或者集合.

\section{1 直和 $\mathscr{D} \dot{+} \boldsymbol{H}(\mathscr{D})$}

引理 2.1 $f \in C[-a, a]$, 其中 $a>0$, 且对任意的 $x \in \mathbb{R} \backslash[-a, a]$ 有 $f(x)=0$. 又设 $f$ 有 $n-1\left(n \in \mathbb{Z}_{+}\right)$阶消失矩, 即 $f$ 满足等式

$$
\int_{\mathbb{R}} t^{k} f(t) d t=0 \quad\left(\forall k \in \mathbb{Z}_{+}, 0 \leqslant k \leqslant n-1\right),
$$

则

$$
\left|x^{n+1} H f(x)-\frac{1}{\pi} \int_{\mathbb{R}} t^{n} f(t) d t\right| \leqslant \frac{1}{\pi} \frac{a^{n+1}}{|x|-a}\|f\|_{1} \quad(\forall|x|>a),
$$

其中 $\|f\|_{1}$ 表示 $f$ 的 $L^{1}(\mathbb{R})$ 范数.

证明 利用 $\operatorname{supp} f \subset[-a, a]$, 有

$$
H f(x)=\frac{1}{\pi} \lim _{A^{-1}, \varepsilon \rightarrow 0} \int_{\varepsilon<|t-x|<A,|t| \leqslant a} \frac{f(t)}{x-t} d t .
$$

对任意 $|x|>a$, 当 $A^{-1}$ 和 $\varepsilon$ 足够小时, 对任意 $|t| \leqslant a$ 有 $\varepsilon<|t-x|<A$, 所以

$$
\{t \in \mathbb{R}|| t \mid \leqslant a\}=\{t \in \mathbb{R}|\varepsilon<| t-x|<A,| t \mid \leqslant a\},
$$

从而

$$
H f(x)=\frac{1}{\pi} \int_{|t| \leqslant a} \frac{f(t)}{x-t} d t=\frac{1}{\pi} \int_{-a}^{a} \frac{f(t)}{x} \sum_{k=0}^{\infty}\left(\frac{t}{x}\right)^{k} d t=\frac{1}{\pi} \int_{-a}^{a} \sum_{k=0}^{\infty} \frac{t^{k} f(t)}{x^{k+1}} d t
$$

对任意 $|x|>a$ 成立. 因为

$$
\sum_{k=0}^{\infty}\left|\frac{t^{k} f(t)}{x^{k+1}}\right|=\frac{|f(t)|}{|x|-|t|} \leqslant \frac{1}{|x|-a}|f(t)| \in L^{1}([-a, a]),
$$

利用 Fubini-Tonelli 定理 ${ }^{[18]}$, 可以得到

$$
H f(x)=\frac{1}{\pi} \int_{-a}^{a} \sum_{k=0}^{\infty} \frac{t^{k} f(t)}{x^{k+1}} d t=\frac{1}{\pi} \sum_{k=0}^{\infty} \frac{1}{x^{k+1}} \int_{-a}^{a} t^{k} f(t) d t,
$$

因此

$$
x^{n+1} H f(x)=\frac{1}{\pi} \sum_{k=0}^{\infty} \frac{1}{x^{k}} \int_{-a}^{a} t^{n+k} f(t) d t
$$

记

$$
y:=\frac{1}{x}, \quad c_{k}:=\int_{-a}^{a} t^{n+k} f(t) d t,
$$


有

$$
\left|x^{n+1} H f(x)-\frac{1}{\pi} \int_{-a}^{a} t^{n} f(t) d t\right| \leqslant \frac{1}{\pi} \sum_{k=1}^{\infty}\left|c_{k} y^{k}\right| \leqslant \frac{1}{\pi} \frac{a^{n+1}}{|x|-a} \int_{-a}^{a}|f(t)| d t \quad(\forall|x|>a) .
$$

引理证毕.

注 1 任意函数都有 -1 阶消失矩, 这是因为在式 $(2.2)$ 中不存在满足 $0 \leqslant k \leqslant-1$ 的整 数 $k$.

注 2 设 $n \in \mathbb{Z}_{+}$, 若函数 $f(x)$ 有 $n-1$ 阶消失矩, 并且满足

$$
\int_{\mathbb{R}} t^{n} \psi(t) d t \neq 0
$$

则称 $f(x)$ 恰有 $n-1$ 阶消失矩.

下面的定理表明线性空间之和 $\mathscr{D}+H(\mathscr{D})$ 是直和, 因而可记为 $\mathscr{D}_{H}=\mathscr{D} \dot{+} H(\mathscr{D})$.

定理 $2.2 \mathscr{D} \cap H(\mathscr{D})=\{0\}$.

证明 若 $\mathscr{D} \cap H(\mathscr{D}) \neq\{0\}$, 则存在非零的 $\phi \in \mathscr{D} \cap H(\mathscr{D})$. 设 $\phi=H \psi$, 其中 $\psi \in \mathscr{D}, \psi \neq \equiv$. 先证明存在 $n \in \mathbb{Z}_{+}$, 使得 $\psi$ 恰有 $n-1$ 阶消失矩.

事实上, 若上述 $n$ 不存在, 设 $\operatorname{supp} \psi \subset[-a, a]$, 其中 $a>0$, 则对任意多项式 $p$, 有

$$
\int_{-a}^{a} p(t) \psi(t) d t=0
$$

考虑到多项式在 $C[-a, a]$ 中稠密, 我们得到 $\psi \equiv 0$, 这与 $\psi \neq \equiv 0$ 矛盾.

对此 $n$, 利用引理 2.1 有

$$
\lim _{x \rightarrow \infty} x^{n+1} \phi(x)=\lim _{x \rightarrow \infty} x^{n+1} H \psi(x)=\frac{1}{\pi} \int_{-a}^{a} t^{n} \psi(t) d t \neq 0 .
$$

这与 $\phi \in \mathscr{D}$ 矛盾. 证毕.

推论 2.3 设 $g \in \mathscr{D}_{H} \backslash \mathscr{D}$. 则存在 $n \in \mathbb{Z}_{+}$以及常数 $c \neq 0$, 使得 $\lim _{x \rightarrow \infty} x^{n+1} g(x)=c$.

证明 $g \in \mathscr{D}_{H} \backslash \mathscr{D}$ 意味着存在 $\phi, \psi \in \mathscr{D}$, 使得 $g=\phi+H \psi$ 且 $\psi \neq \equiv 0$. 于是存在 $n \in \mathbb{Z}_{+}$, 使 得 $\psi$ 恰有 $n-1\left(n \in \mathbb{Z}_{+}\right)$阶消失矩. 利用引理 2.1, 存在常数 $c \neq 0$, 使得 $\lim _{x \rightarrow \infty} x^{n+1} H \psi(x)=$ $c$, 于是

$$
\lim _{x \rightarrow \infty} x^{n+1} g(x)=\lim _{x \rightarrow \infty} x^{n+1}[\phi(x)+H \psi(x)]=c .
$$

证毕.

\section{$2.2 \mathscr{D}_{H}$ 中的拓扑}

由于 $\mathscr{D}_{H}:=\mathscr{D} \dot{+} H(\mathscr{D})$ 是直和, 我们定义 $\mathscr{D}_{H}$ 中的收玫性如下: $\forall\left\{\phi_{n}+H \psi_{n}\right\} \subset \mathscr{D}_{H}$, 若在 $\mathscr{D}$ 中有 $\phi_{n}, \psi_{n} \rightarrow 0$, 则在 $\mathscr{D}_{H}$ 中定义 $\phi_{n}+H \psi_{n} \rightarrow 0$. 式 $(2.3)$ 被赋予上述拓扑后, 空间 $\mathscr{D}_{H}$ 就成 为一个满足 $H\left(\mathscr{D}_{H}\right)=\mathscr{D}_{H}$ 的拓扑向量空间, 且 $H: \mathscr{D}_{H} \rightarrow \mathscr{D}_{H}$ 是一个连续线性算子. 又因为 $H^{-1}=-H$, 从而 $H$ 是 $\mathscr{D}_{H}$ 上的同胚映射.

设 $\mathscr{X}$ 和 $\mathscr{Y}$ 是两个拓扑向量空间, 且 $\mathscr{X} \subset \mathscr{Y}$. 若对任意 $\left\{x_{n}\right\} \subset \mathscr{X},\left\{x_{n}\right\}$ 在 $\mathscr{X}$ 中收玫 到 0 蕴含 $\left\{x_{n}\right\}$ 在 $\mathscr{Y}$ 中收玫到 0 , 则称空间 $\mathscr{X}$ 连续嵌入到空间 $\mathscr{Y}$, 并记为 $\mathscr{X} \subset \mathscr{Y}^{[17]}$.

众所周知, 对 $\mathscr{X}$ 的对偶空间 $\mathscr{X}^{\prime}$ 赋予通常的加法, 数乘以及如下的收玫性后成为拓扑 向量空间: $\mathscr{X}^{\prime}$ 中序列 $\left\{f_{n}\right\}$ 收玫到 0 是指 $f_{n}(x) \rightarrow 0$ 对任意 $x \in \mathscr{X}$ 成立. 易证 $\mathscr{X} \subset \mathscr{Y}$ 蕴 含 $\mathscr{Y}^{\prime} \subset \mathscr{X}^{\prime}$. 
对于上面定义的拓扑向量空间 $\mathscr{D}_{H}$, 易证有 $\mathscr{D} \subset \mathscr{D}_{H}$, 从而 $\mathscr{D}_{H}^{\prime} \subseteq \mathscr{D}^{\prime}$. 接下来的定理进一 步表明: $\mathscr{D}_{H}$ 是满足 $\mathscr{D} \subset \mathscr{D}_{H}$ 且使得 $H$ 为映 $\mathscr{D}_{H}$ 到自身的同胚映射的最小空间.

定理 2.4 设 $\mathscr{X}$ 是一个拓扑向量空间且满足 $\mathscr{D} \subset \mathscr{X} \subset L^{2}(\mathbb{R})$, 若 Hilbert 变换 $H$ : $\mathscr{X} \rightarrow \mathscr{X}$ 是一个连续线性算子. 则有 $\mathscr{D}_{H} \subset \mathscr{X}$, 从而 $\mathscr{X}^{\prime} \subset \mathscr{D}_{H}^{\prime}$.

证明 连续嵌入关系 $\mathscr{D} \subset \mathscr{X}$ 蕴含了 $H(\mathscr{D}) \subset H(\mathscr{X}) \subset \mathscr{X}$, 因此 $\mathscr{D}_{H} \subset \mathscr{X}$.

对任意 $\left\{\phi_{n}+H \psi_{n}\right\} \subset \mathscr{D}_{H}$, 若在 $\mathscr{D}_{H}$ 中有 $\phi_{n}+H \psi_{n} \rightarrow 0$, 则在 $\mathscr{D}$ 中有 $\phi_{n}, \psi_{n} \rightarrow 0$. 于 是在 $\mathscr{X}$ 中有 $\phi_{n}, \psi_{n} \rightarrow 0$, 由 $H$ 的连续性有 $H \psi_{n} \rightarrow 0$. 所以在 $\mathscr{X}$ 中有 $\phi_{n}+H \psi_{n} \rightarrow 0$, 这表 明 $\mathscr{D}_{H} \subset \mathscr{X}$. 证毕.

对 $1<p<\infty$, 记 $\mathscr{D}_{L^{p}}:=\left\{f \mid f \in C^{\infty}(\mathbb{R})\right.$ 且 $\left.f^{(k)} \in L^{p}(\mathbb{R})\left(\forall k \in \mathbb{Z}_{+}\right)\right\}$. 在文献 $[14,15]$ 中已经证明了 $H: \mathscr{D}_{L^{p}} \rightarrow \mathscr{D}_{L^{p}}$ 是连续线性算子. 利用定理 2.4, 可以得到 $\mathscr{D}_{H} \subset \mathscr{D}_{L^{p}}$. 上述 式子蕴含 $\mathscr{D}_{L^{p}}^{\prime} \subset \mathscr{D}_{H}^{\prime}$ 且 $\mathscr{D}_{H} \subset \bigcap_{1<p<\infty} \mathscr{D}_{L^{p}}$. 下面证明 $\mathscr{D}_{H} \varsubsetneqq \bigcap_{1<p<\infty} \mathscr{D}_{L^{p}}$. 事实上, Gauss 函数 $g(x):=e^{-|x|^{2}}$ 属于 $\bigcap_{1<p<\infty} \mathscr{D}_{L^{p}}$, 且对任意 $n \in \mathbb{N}$, 满足 $\lim _{x \rightarrow \infty} x^{n} g(x)=0$, 这表明 $g \notin \mathscr{D}_{H} \backslash \mathscr{D}$; 另一方面, 显然有 $g \notin \mathscr{D}$, 因此, $g \notin \mathscr{D}_{H}$.

例 1 设 $x \in \mathbb{R}$. 定义 Dirac 脉冲函数 $\delta_{x}$ 如下: $\delta_{x}(\phi):=\phi(x)\left(\forall \phi \in \mathscr{D}_{H}\right)$, 则 $\delta_{x} \in \mathscr{D}_{H}^{\prime}$.

证明 对任意 $g=\phi+H \psi \in \mathscr{D}_{H}, \phi, \psi \in \mathscr{D}$, 显然 $\left\langle\delta_{x}, g\right\rangle:=g(x)=\phi(x)+(H \psi)(x)$ 是 $\mathscr{D}_{H}$ 上的线性泛函. 为了证明它的连续性, 只要证明对任意 $\left\{\psi_{n}\right\} \subset \mathscr{D}$ 且 $\psi_{n} \rightarrow 0$, 在 $\mathscr{D}$ 中有 $\delta_{x}\left(H \psi_{n}\right)=\left(H \psi_{n}\right)(x) \rightarrow 0$.

对任意 $\left\{\psi_{n}\right\} \subset \mathscr{D}$ 且 $\psi_{n} \rightarrow 0$, 存在 $a>0$, 使得 $\operatorname{supp} \psi_{n} \subset[-a, a]$. 利用下一节的引理 3.1, 有

$$
\begin{aligned}
\left|H \psi_{n}(x)\right| & \leqslant(1+|x|)\left|H \psi_{n}(x)\right| \leqslant C(a)\left(\left\|\psi_{n}\right\|_{2}+\left\|\psi_{n}^{\prime}\right\|_{2}\right) \\
& \leqslant C(a) \sqrt{2 a}\left(\left\|\psi_{n}^{\prime}\right\|_{C(\mathbb{R})}+\left\|\psi_{n}\right\|_{C(\mathbb{R})}\right) \rightarrow 0 \quad(n \rightarrow \infty),
\end{aligned}
$$

所以, $\delta_{x} \in \mathscr{D}_{H}^{\prime}$. 证毕.

\section{3 分布空间 $\mathscr{D}_{H}^{\prime}$ 中的嵌人定理}

如定理 2.4 所述, $\mathscr{D}_{H}$ 是满足 $\mathscr{D} \subset \mathscr{D}_{H}$ 并且使得 $H$ 在 $\mathscr{D}_{H}$ 上是同胚映射的最小空间, 即 其对偶空间 $\mathscr{D}_{H}^{\prime}$ 是最大的空间. 本节将证明许多常用的空间都可连续嵌入到 $\mathscr{D}_{H}^{\prime}$ 中.

\section{1 一般嵌人定理}

引理 3.1. 设 $\psi \in \mathscr{D}$ 有 $n-1$ 阶消失矩且 $\operatorname{supp} \psi \subset[-a, a]$, 其中 $n$ 是某个非负整数, $a>0$, 则存在常数 $C(a, n)>0$, 使得下面的不等式成立:

$$
\left(1+|x|^{n+1}\right)|H \psi(x)| \leqslant C(a, n)\|\psi\|_{1,2} \quad(\forall x \in \mathbb{R}),
$$

其中 $\|\psi\|_{1,2}:=\|\psi\|_{2}+\left\|\psi^{\prime}\right\|_{2}$, 而 $\|\cdot\|_{2}$ 表示通常的 $L^{2}(\mathbb{R})$ 范数.

证明 不失一般性, 假设 $\psi \neq \equiv$. 根据引理 2.1 , 有

$$
\left|x^{n+1} H \psi(x)-\frac{1}{\pi} \int_{-a}^{a} t^{n} \psi(t) d t\right| \leqslant \frac{1}{\pi} \frac{a^{n+1}}{|x|-a}\|\psi\|_{1} \quad(\forall|x|>a),
$$

于是得到

$$
\left|x^{n+1} H \psi(x)\right| \leqslant \frac{1}{\pi} a^{n}\left(1+\frac{a}{|x|-a}\right)\|\psi\|_{1} \leqslant C(a, n)\|\psi\|_{2} \quad(\forall|x|>2 a) .
$$

为估计 $|x| \leqslant 2 a$ 时的 $|H \psi(x)|$, 设 $H \psi$ 在点 $\xi \in[-2 a, 2 a]$ 处达到其在 $[-2 a, 2 a]$ 上的最小 
值, 那么有 ${ }^{[15]}$

$$
|H \psi(x)-H \psi(\xi)|=\left|\int_{\xi}^{x}(H \psi)^{\prime}(t) d t\right|=\left|\int_{\xi}^{x} H \psi^{\prime}(t) d t\right| \leqslant \sqrt{4 a}\left\|H \psi^{\prime}\right\|_{2}=2 \sqrt{a}\left\|\psi^{\prime}\right\|_{2} .
$$

然而

$$
|H \psi(\xi)| \leqslant\left(\frac{1}{4 a} \int_{-2 a}^{2 a}|H \psi(t)|^{2} d t\right)^{1 / 2} \leqslant \frac{1}{2 \sqrt{a}}\|H \psi\|_{2}=\frac{1}{2 \sqrt{a}}\|\psi\|_{2},
$$

所以

$$
|H \psi(x)| \leqslant|H \psi(x)-H \psi(\xi)|+|H \psi(\xi)| \leqslant C(a)\left(\left\|\psi^{\prime}\right\|_{2}+\|\psi(t)\|_{2}\right) \quad(\forall|x| \leqslant 2 a) .
$$

证毕.

定理 3.2. 设 $\frac{f}{1+|\cdot|^{2}} \in L^{1}(\mathbb{R})$, 且极限

$$
\lim _{A \rightarrow \infty} \int_{1}^{A} \frac{f(x)}{x} d x, \quad \lim _{A \rightarrow \infty} \int_{-A}^{-1} \frac{f(x)}{x} d x
$$

存在, 定义泛函 $F_{f}$ 如下:

$$
F_{f}(\phi):=\langle f, \phi\rangle:=\lim _{A, B \rightarrow \infty} \int_{-A}^{B} f(x) \phi(x) d x \quad\left(\forall \phi \in \mathscr{D}_{H}\right),
$$

则 $f \in \mathscr{D}_{H}^{\prime}, f$ 被 $F_{f}$ 唯一决定, 且对任意 $a \geqslant 1$, 有 其中

$$
\begin{cases}\left|F_{f}(\phi)\right| \leqslant C(a)\|\phi\|_{1,2} \int_{\mathbb{R}} \frac{|f(x)|}{1+x^{2}} d x, & \forall \phi \in \mathscr{D}, \operatorname{supp} \phi \subset[-a, a], \\ \left|F_{f}(H \psi)\right| \leqslant C(a)\|\psi\|_{1,2}\left[\left|\lambda_{f}(2 a)\right|+\int_{\mathbb{R}} \frac{|f(x)|}{1+x^{2}} d x\right], & \forall \psi \in \mathscr{D}, \operatorname{supp} \psi \subset[-a, a],\end{cases}
$$

$$
\lambda_{f}(2 a):=\lim _{A, B \rightarrow \infty} \int_{[-A, B] \backslash[-2 a, 2 a]} \frac{f(x)}{x} d x .
$$

证明 易证 $f$ 在任何有界区间上是 Lebesgue 可积的, 于是对任意 $\phi \in \mathscr{D}$, 有 $f \phi \in L^{1}(\mathbb{R})$, 从而式 (3.2) 右边的极限存在, 且容易证得

$$
\left|F_{f}(\phi)\right| \leqslant\left(\int_{\mathbb{R}} \frac{|f(x)|}{1+x^{2}} d x\right) \max _{x \in \operatorname{supp} \phi}\left[\left(1+x^{2}\right)|\phi(x)|\right] \quad(\forall \phi \in \mathscr{D}) .
$$

对任意 $a \geqslant 1$, 设 $\phi \in \mathscr{D}, \operatorname{supp} \phi \subset[-a, a]$ 且 $|\phi|$ 在点 $\xi \in[-a, a]$ 达到最小值. 我们有

$$
|\phi(\xi)| \leqslant C(a)\|\phi\|_{2} \quad \text { 且 }|\phi(x)-\phi(\xi)|=\left|\int_{x}^{\xi} \phi^{\prime}(t) d t\right| \leqslant C(a)\left\|\phi^{\prime}\right\|_{2} \quad(\forall|x| \leqslant a),
$$

从而 $|\phi(x)| \leqslant C(a)\left(\|\phi\|_{2}+\left\|\phi^{\prime}\right\|_{2}\right)(\forall|x| \leqslant a)$, 因此

$$
\left|F_{f}(\phi)\right| \leqslant C(a)\|\phi\|_{1,2} \int_{\mathbb{R}} \frac{|f(x)|}{1+x^{2}} d x \quad(\forall \phi \in \mathscr{D}) .
$$

式 (3.3) 之第 1 个不等式得证.

设 $\psi \in \mathscr{D}$ 且 $\operatorname{supp} \psi \subset[-a, a]$, 则 $\phi=H \psi \in H(\mathscr{D})$, 以下考虑式 (3.2) 右边极限的存在性. 根据引理 2.1 , 有

$$
\left|x H \psi(x)-c_{\psi}\right| \leqslant \frac{1}{\pi} \frac{a}{|x|-a}\|\psi\|_{1} \leqslant \frac{C(a)}{|x|}\|\psi\|_{2} \quad(\forall|x| \geqslant 2 a),
$$

其中 $c_{\psi}:=\frac{1}{\pi} \int_{-a}^{a} \psi(t) d t$. 所以

$$
\left|\frac{f(x)}{x}\left[x H \psi(x)-c_{\psi}\right]\right| \leqslant C(a) \frac{|f(x)|}{x^{2}}\|\psi\|_{2} \in L^{1}(\mathbb{R} \backslash[-2 a, 2 a]) .
$$


因此

$$
\lim _{A, B \rightarrow \infty} \int_{[-A, B] \backslash[-2 a, 2 a]} \frac{f(x)}{x}\left[x H \psi(x)-c_{\psi}\right] d x=\int_{\mathbb{R} \backslash[-2 a, 2 a]} \frac{f(x)}{x}\left[x H \psi(x)-c_{\psi}\right] d x,
$$

从而

$$
\lim _{A, B \rightarrow \infty} \int_{[-A, B] \backslash[-2 a, 2 a]} f(x) H \psi(x) d x=c_{\psi} \lambda_{f}(2 a)+\int_{\mathbb{R} \backslash[-2 a, 2 a]} \frac{f(x)}{x}\left[x H \psi(x)-c_{\psi}\right] d x .
$$

所以, 式 (3.2) 右边的极限存在, 并且有

$$
F_{f}(H \psi)=c_{\psi} \lambda_{f}(2 a)+\int_{\mathbb{R} \backslash[-2 a, 2 a]} \frac{f(x)}{x}\left[x H \psi(x)-c_{\psi}\right] d x+\int_{-2 a}^{2 a} f(x) H \psi(x) d x .
$$

利用引理 3.1 有

$$
\int_{-2 a}^{2 a}|f(x) H \psi(x)| d x \leqslant C(a)\|\psi\|_{1,2} \int_{\mathbb{R}} \frac{|f(x)|}{1+x^{2}} d x,
$$

所以

$$
\left|F_{f}(H \psi)\right| \leqslant\left|c_{\psi} \lambda_{f}(2 a)\right|+C(a)\|\psi\|_{1,2} \int_{\mathbb{R} \backslash[-2 a, 2 a]} \frac{|f(x)|}{1+x^{2}} d x .
$$

联立上式与不等式 $\left|c_{\psi}\right| \leqslant C(a)\|\psi\|_{2}$ 便知式 (3.3) 的第 2 个不等式成立.

易见 $F_{f}$ 是 $\mathscr{D}_{H}$ 上的一个线性泛函, 利用式 (3.3) 易证 $F_{f}$ 在 $\mathscr{D}_{H}$ 上的连续性. 这便证明 了 $F_{f} \in \mathscr{D}_{H}^{\prime}$.

最后, 由 $f \in L_{\mathrm{loc}}(\mathbb{R})$ 和 $\mathscr{D} \subset \mathscr{D}_{H}$ 易知 $f$ 由 $F_{f}$ 唯一确定. 证毕.

注 1 通常, 在不致引起混淆的情形下, 我们把式 (3.2) 中定义的泛函 $F_{f}$ 记为 $f$, 亦将 $F_{f}(\phi)$ 记为 $\langle f, \phi\rangle$, 因而有 $f \in \mathscr{D}_{H}^{\prime}$.

注 2 若 $\frac{f}{1+|\cdot|} \in L^{1}(\mathbb{R})$, 则定理 3.2 中的条件都满足, 此时式 (3.2) 中定义的泛函 $F_{f}$ 可 表示为

$$
F_{f}(\phi):=\langle f, \phi\rangle=\int_{\mathbb{R}} f(x) \phi(x) d x \quad\left(\forall \phi \in \mathscr{D}_{H}\right) .
$$

下面考虑定理 3.2 的另一充分条件. 首先把 Riemann 积分之第二均值定理推广至 Lebegues 积分.

引理 3.3 设 $f \in L^{1}([a, b]), g$ 是 $[a, b]$ 上的单调函数, 则存在点 $\xi \in[a, b]$, 使得

$$
\int_{a}^{b} f(x) g(x) d x=g(a) \int_{a}^{\xi} f(x) d x+g(b) \int_{\xi}^{b} f(x) d x .
$$

证明 根据 $C[a, b]$ 在 $L^{1}([a, b])$ 中的稠密性以及 Riemann 积分的第二均值定理, 可容易 地证明该引理. 这里我们省去其证明.

推论 3.4 设 $\frac{f}{1+|\cdot|^{2}} \in L^{1}(\mathbb{R})$. 若存在常数 $C \geqslant 0$, 使得下面两个不等式成立:

$$
\left|\int_{1}^{A} f(x) d x\right| \leqslant C, \quad\left|\int_{-A}^{-1} f(x) d x\right| \leqslant C \quad(\forall A \geqslant 1),
$$

则定理 3.2 的结论成立.

证明 对任意的 $A_{2} \geqslant A_{1} \geqslant 1$, 由引理 3.3 , 可知存在 $\xi \in\left[A_{1}, A_{2}\right]$, 使得

$$
\left|\int_{A_{1}}^{A_{2}} \frac{f(x)}{x} d x\right|=\left|\frac{1}{A_{1}} \int_{A_{1}}^{\xi} f(x) d x+\frac{1}{A_{2}} \int_{\xi}^{A_{2}} f(x) d x\right| \leqslant C\left(\frac{1}{A_{1}}+\frac{1}{A_{2}}\right) \rightarrow 0 \quad\left(A_{1}, A_{2} \rightarrow \infty\right),
$$

因而 $\lim _{A \rightarrow \infty} \int_{1}^{A} \frac{f(x)}{x} d x$ 存在. 类似地, $\lim _{A \rightarrow \infty} \int_{-A}^{-1} \frac{f(x)}{x} d x$ 也存在. 根据定理 3.2, 推论获证. 
3.2 嵌人: $L^{p}(\mathbb{R}) \subset \mathscr{D}_{H}^{\prime}(1 \leqslant p<\infty)$

以下的推论说明 $L^{p}(\mathbb{R})$ 连续嵌入到 $\mathscr{D}_{H}^{\prime}$.

推论 3.5 $L^{p}(\mathbb{R}) \subset \mathscr{D}_{H}^{\prime}(1 \leqslant p<\infty)$.

证明 设 $f \in L^{p}(\mathbb{R})$, 则有 $\frac{f}{1+|\cdot|} \in L^{1}(\mathbb{R})$, 于是 $L^{p}(\mathbb{R}) \subset \mathscr{D}_{H}^{\prime}$.

令 $\left\{f_{n}\right\} \subset L^{p}(\mathbb{R})$ 满足 $\left\|f_{n}\right\|_{p} \rightarrow 0(n \rightarrow \infty)$.

(i) 对任意 $\phi \in \mathscr{D}$, 设 $\operatorname{supp} \phi \subset[-a, a]$. 由式 (3.3) 有

$$
\left|\left\langle f_{n}, \phi\right\rangle\right| \leqslant C(a)\|\phi\|_{1,2} \int_{\mathbb{R}} \frac{\left|f_{n}(x)\right|}{1+x^{2}} d x \leqslant C(a, p, \phi)\left\|f_{n}\right\|_{p} \rightarrow 0 \quad(n \rightarrow \infty) .
$$

(ii) 对任意 $\psi \in \mathscr{D}$, 设 $\operatorname{supp} \psi \subset[-a, a]$. 类似地, 由式 (3.3) 有

$$
\left|\left\langle f_{n}, H \psi\right\rangle\right| \leqslant C(a, p, \psi)\left[\left|\lambda_{f_{n}}(2 a)\right|+\left\|f_{n}\right\|_{p}\right] \rightarrow 0 \quad(n \rightarrow \infty),
$$

其中

$$
\left|\lambda_{f_{n}}(2 a)\right|=\lim _{A, B \rightarrow \infty}\left|\int_{[-A, B] \backslash[-2 a, 2 a]} \frac{f_{n}(x)}{x} d x\right| \leqslant C(a, p)\left\|f_{n}\right\|_{p} .
$$

推论获证.

3.3 嵌入: $\dot{L}_{T}^{1} \subset \mathscr{D}_{H}^{\prime}$

设 $L_{T}^{p}(1 \leqslant p<\infty, T>0)$ 表示以 $T$ 为周期且满足

$$
\|f\|_{L_{T}^{p}}:=\left(\int_{0}^{T}|f(x)|^{p} d x\right)^{1 / p}<\infty
$$

的所有 Lebesgue 可测函数所构成的空间. 记

$$
\dot{L}_{T}^{p}:=\left\{f \in L_{T}^{p} \mid \int_{0}^{T} f(x) d x=0\right\} .
$$

我们有如下的嵌入结果:

推论 3.6 $\dot{L}_{T}^{1} \subset \mathscr{D}_{H}^{\prime}$.

证明 对任意 $f \in \dot{L}_{T}^{1}$, 易证 $\frac{f}{1+|\cdot|^{2}} \in L^{1}(\mathbb{R})$. 对 $A \geqslant 1$, 取 $k \in \mathbb{Z}_{+}$满足 $1+k T \leqslant A<$ $1+(k+1) T$, 则有

$$
\left|\int_{1}^{A} f(x) d x\right|=\left|\left(\int_{1}^{1+k T}+\int_{1+k T}^{A}\right) f(x) d x\right| \leqslant \int_{1+k T}^{A}|f(x)| d x \leqslant\|f\|_{L_{T}^{1}} .
$$

类似地, 有 $\left|\int_{-A}^{-1} f(x) d x\right| \leqslant\|f\|_{L_{T}^{1}}$. 利用推论 3.4, 有 $f \in \mathscr{D}_{H}^{\prime}$, 所以 $\dot{L}_{T}^{1} \subset \mathscr{D}_{H}^{\prime}$.

为证明 $\dot{L}_{T}^{1}$ 连续嵌入到 $\mathscr{D}_{H}^{\prime}$, 我们令 $\left\{f_{n}\right\} \subset \dot{L}_{T}^{1}$ 且满足 $\left\|f_{n}\right\|_{L_{T}^{1}} \rightarrow 0(n \rightarrow \infty)$. 设 $\forall \phi, \psi \in \mathscr{D}$ 满足 $\operatorname{supp} \phi, \operatorname{supp} \psi \subset[-a, a]$, 由式 (3.3), 有

$$
\left|\left\langle f_{n}, \phi\right\rangle\right| \leqslant C(a)\|\phi\|_{1,2}\left\|f_{n}\right\|_{L_{T}^{1}} \rightarrow 0 \quad(n \rightarrow \infty),
$$

且

$$
\left|\left\langle f_{n}, H \psi\right\rangle\right| \leqslant C(a)\|\psi\|_{1,2}\left[\left|\lambda_{f_{n}}(2 a)\right|+\left\|f_{n}\right\|_{L_{T}^{1}}\right]
$$

其中

$$
\left|\lambda_{f_{n}}(2 a)\right|=\lim _{A, B \rightarrow \infty}\left|\int_{[-A, B] \backslash[-2 a, 2 a]} \frac{f_{n}(x)}{x} d x\right| .
$$

对任意 $B \geqslant 2 a$, 根据引理 3.3 , 存在 $\xi_{n} \in[2 a, B]$, 使得

$$
\int_{2 a}^{B} \frac{f_{n}(x)}{x} d x=\frac{1}{2 a} \int_{\xi_{n}}^{B} f_{n}(x) d x+\frac{1}{B} \int_{\xi_{n}}^{B} f_{n}(x) d x .
$$


类似式 (3.8) 的证明, 有 $\left|\int_{2 a}^{B} \frac{f_{n}(x)}{x} d x\right| \leqslant \frac{1}{a}\left\|f_{n}\right\|_{L_{T}^{1}}$. 同样地, 对 $\forall A \geqslant 2 a$, 有 $\left|\int_{-A}^{-2 a} \frac{f_{n}(x)}{x} d x\right| \leqslant$ $\frac{1}{a}\left\|f_{n}\right\|_{L_{T}^{1}}$. 所以 $\left|\lambda_{f_{n}}(2 a)\right| \leqslant \frac{2}{a}\left\|f_{n}\right\|_{L_{T}^{1}}$. 联立式 (3.9) 得到 $\left|\left\langle f_{n}, H \psi\right\rangle\right| \leqslant C(a)\|\psi\|_{1,2}\left\|f_{n}\right\|_{L_{T}^{1}} \rightarrow$ $0(n \rightarrow \infty)$. 证毕.

例 2 设 $f(x)=\cos \omega x$ 或者 $\sin \omega x$, 其中 $\omega \in \mathbb{R} \backslash\{0\}$, 则有 $f \in \mathscr{D}_{H}^{\prime}$.

\section{4 非线性相位信号 $\{\cos \theta(x), \sin \theta(x)\} \subset \mathscr{D}_{H}^{\prime}$}

前两个小节证明了经典函数空间 $L^{p}(\mathbb{R})$ 和 $\dot{L}_{T}^{1}$ 是 $\mathscr{D}_{H}^{\prime}$ 的子空间. 然而在信号处理中经常 遇到一些信号, 如线性鸟鸣声 $s(t)=\cos \left(b t^{2}+c t\right.$ ) (其中 $b, c \in \mathbb{R}$ ) 就不在这两个函数空间中. 在 这个小节中, 我们将证明 $\mathscr{D}_{H}^{\prime}$ 包含了一类形如 $\cos \theta(x)$ 和 $\sin \theta(x)$ 的非线性相位信号, 从而使 得时频分析和信号处理中所遇到的几乎所有的信号都属于 $\mathscr{D}_{H}^{\prime}$.

定理 3.7 若 $\theta \in C^{1}(\mathbb{R})$, 存在常数 $A>0$, 使得 $\theta$ 在 $(-\infty,-A)$ 和 $(A, \infty)$ 上分别是严 格单调函数, 且 $\lim _{|x| \rightarrow \infty}|\theta(x)|=\infty$, 则 $\cos \theta(\cdot), \sin \theta(\cdot) \in \mathscr{D}_{H}^{\prime}$.

证明 易见 $\frac{\cos \theta(\cdot)}{1+|\cdot|^{2}} \in L^{1}(\mathbb{R})$. 不失一般性, 假设 $\theta$ 在 $[1, \infty)$ 上严格递增. 令 $\left\{x_{k}\right\}_{k=1}^{\infty}$ 满足 $\theta\left(x_{k}\right)=k \pi+\frac{\pi}{2}(\forall k \in \mathbb{N})$, 则对 $\forall n, m \in \mathbb{N}, n>m$, 有

$$
\begin{aligned}
\int_{x_{m}}^{x_{n}} \frac{\cos \theta(x)}{x} d x & =\int_{m \pi+\pi / 2}^{n \pi+\pi / 2}\left[\ln \theta^{-1}(t)\right]^{\prime} \cos t d t \\
& =\int_{m \pi+\pi / 2}^{n \pi+\pi / 2}\left[\ln \theta^{-1}(t)\right] \sin t d t \\
& =\sum_{k=m+1}^{n}(-1)^{k} \int_{0}^{\pi / 2} \ln \frac{\theta^{-1}(k \pi+t)}{\theta^{-1}(k \pi-t)} \sin t d t .
\end{aligned}
$$

不难验证 $\ln \theta^{-1}(t)$ 严格递增且满足 $\lim _{|t| \rightarrow \infty}\left|\theta^{-1}(t)\right|=\infty$, 所以

$$
\begin{aligned}
\left|\int_{x_{m}}^{x_{n}} \frac{\cos \theta(x)}{x} d x\right| & \leqslant \sum_{k=m+1}^{n} \int_{0}^{\pi / 2} \ln \frac{\theta^{-1}(k \pi+t)}{\theta^{-1}(k \pi-t)} \sin t d t \\
& \leqslant \sum_{k=m+1}^{n} \ln \frac{\theta^{-1}\left(k \pi+\frac{1}{2} \pi\right)}{\theta^{-1}\left(k \pi-\frac{1}{2} \pi\right)} \\
& =\ln \theta^{-1}\left(n \pi+\frac{1}{2} \pi\right)-\ln \theta^{-1}\left(m \pi+\frac{1}{2} \pi\right) \rightarrow 0 \quad(n, m \rightarrow \infty),
\end{aligned}
$$

这表明 $\lim _{A \rightarrow \infty} \int_{1}^{A} \frac{\cos \theta(x)}{x} d x$ 存在. 类似地可以证明 $\lim _{A \rightarrow \infty} \int_{-A}^{-1} \frac{\cos \theta(x)}{x} d x$ 也存在. 根据 定理 3.2 , 有 $\cos \theta(x) \in \mathscr{D}_{H}^{\prime}$. 同理可证 $\sin \theta(x) \in \mathscr{D}_{H}^{\prime}$. 证毕

利用本节所得结果, 容易验证在时频分析和信号处理中所用到的几乎所有的信号都属于 $\mathscr{D}_{H}^{\prime}$. 以下的信号来自文献 [2].

例 3 以下的所有信号都属于 $\mathscr{D}_{H}^{\prime}$ :

(1) 线性鸟鸣声 $s(t)=\exp \left(i \beta t^{2}+i \gamma t\right)$, 其中 $\beta \neq 0$;

(2) Gauss 包络: $s(t)=\exp \left(-\alpha t^{2}+i \beta t^{2}+i \gamma t\right)$, 其中 $\alpha>0$;

(3) $s(t)=\exp \left(-\alpha t^{2}+i \beta t^{2}+i m \sin \left(\omega_{m} t\right)+i \omega_{0} t\right)$, 其中 $\alpha>0$;

(4) $s(t)=\exp \left(i \beta t^{2}+i m \sin \left(\omega_{m} t\right)+i \omega_{0} t\right)$, 其中 $\beta \neq 0$.

证明 根据定理 3.7, 易证 (1) 和 (4) 中的信号均属于 $\mathscr{D}_{H}^{\prime}$ : 对任意 $\alpha>0$, 由于 (2) 和 (3) 中的信号 $s(t)$ 都属于 $L^{2}(\mathbb{R})$, 故在 $\mathscr{D}_{H}^{\prime}$ 中. 


\section{4 分布的 Hilbert 变换}

\section{1 利用共轭算子延拓 Hilbert 变换}

本节利用共轭算子的方法将经典的 Hilbert 变换 $H$ 延拓到空间 $\mathscr{D}_{H}^{\prime}$ 上. 我们先来回顾一 下下面的等式 (参见文献 $[19$, p. 132]):

$$
\int_{\mathbb{R}}(H f)(x) \phi(x) d x=-\int_{\mathbb{R}} f(x)(H \phi)(x) d x \quad\left(\forall f, \phi \in L^{2}(\mathbb{R})\right) .
$$

考虑 $H$ 在 $\mathscr{D}_{H}$ 上的限制, 我们知道 $H: \mathscr{D}_{H} \rightarrow \mathscr{D}_{H}$ 是连续线性算子. $\forall f \in \mathscr{D}_{H}^{\prime}, \phi \in \mathscr{D}_{H}$, $H$ 的共轭算子 $H^{*}$ 定义为 $\left\langle H^{*} f, \phi\right\rangle:=\langle f, H \phi\rangle . H$ 的连续线性性蕴含了共轭算子 $H^{*}: \mathscr{D}_{H}^{\prime} \rightarrow$ $\mathscr{D}_{H}^{\prime}$, 也是连续线性算子. 对任意 $f \in \mathscr{D}_{H}$, 利用式 (4.1), 有

$$
\langle H f, \phi\rangle:=\int_{\mathbb{R}}(H f)(x) \phi(x) d x=-\int_{\mathbb{R}} f(x)(H \phi)(x) d x=-\langle f, H \phi\rangle=\left\langle-H^{*} f, \phi\right\rangle \quad\left(\forall \phi \in \mathscr{D}_{H}\right),
$$

也就是说, 在 $\mathscr{D}_{H}^{\prime}$ 中有 $H f=-H^{*} f$. 若 $S: \mathscr{D}_{H} \rightarrow \mathscr{D}_{H}$ 也是连续线性算子, 并且满足 $H f=S^{*} f\left(\forall f \in \mathscr{D}_{H}\right)$, 则

$$
\int_{\mathbb{R}} f(x)(H \phi)(x) d x=-\int_{\mathbb{R}} f(x)(S \phi)(x) d x \quad\left(\forall f, \phi \in \mathscr{D}_{H}\right),
$$

由此得出 $S \phi=-H \phi\left(\forall \phi \in \mathscr{D}_{H}\right)$, 因此 $-H^{*}$ 可以认为是 $H$ 在分布空间 $\mathscr{D}_{H}^{\prime}$ 上的延拓.

定义 4.1 设 $H^{*}: \mathscr{D}_{H}^{\prime} \rightarrow \mathscr{D}_{H}^{\prime}$ 是经典 Hilbert 变换 $H: \mathscr{D}_{H} \rightarrow \mathscr{D}_{H}$ 的共轭算子, 则 $-H^{*}: \mathscr{D}_{H}^{\prime} \rightarrow \mathscr{D}_{H}^{\prime}$ 定义为 $H$ 到分布空间 $\mathscr{D}_{H}^{\prime}$ 上的推广, 在不致引起混淆的情形下, 将 $-H^{*}$ 简 记为 $H$.

易证推广的 Hilbert 变换把空间 $\mathscr{D}_{H}^{\prime}$ 同胚映射到自身.

定理 $4.2 \quad H: \mathscr{D}_{H}^{\prime} \rightarrow \mathscr{D}_{H}^{\prime}$ 满足 $-H^{2}=I$ (恒等算子).

证明 对任意 $f \in \mathscr{D}_{H}^{\prime}$, 有

$$
\left\langle H^{2} f, \phi\right\rangle=-\langle H f, H \phi\rangle=\left\langle f, H^{2} \phi\right\rangle=-\langle f, \phi\rangle \quad\left(\forall \phi \in \mathscr{D}_{H}\right),
$$

故有 $-H^{2}=I$.

\section{2 经典 Hilbert 变换与推广的 Hilbert 变换的一致性}

定理 4.3 令 $f \in L^{p}(\mathbb{R})(1<p<\infty)$, 则经典的 Hilbert 变换 $H f$ 与在定义 4.1 中推广 的 Hilbert 变换是一致的.

证明 根据式 (3.5), 经典 Hilbert 变换 $H f$ 可以认为是空间 $\mathscr{D}_{H}$ 中的一个分布, 它在 $\phi \in \mathscr{D}_{H}$ 上的作用如下:

$$
\langle H f, \phi\rangle=\int_{\mathbb{R}}(H f)(x) \phi(x) d x .
$$

另一方面, 作为推广的 Hilbert 变换, $H f$ 也可以看作是空间 $\mathscr{D}_{H}$ 上的一个分布, 它在 $\phi \in \mathscr{D}_{H}$ 上的作用如下:

$$
\langle H f, \phi\rangle=-\left\langle H^{*} f, \phi\right\rangle=-\langle f, H \phi\rangle=-\int_{\mathbb{R}} f(x)(H \phi)(x) d x
$$

由于

$$
\int_{\mathbb{R}}(H f)(x) \phi(x) d x=-\int_{\mathbb{R}} f(x)(H \phi)(x) d x \quad\left(\forall f \in L^{p}(\mathbb{R}), \phi \in \mathscr{D}_{H}\right),
$$

故式 (4.2) 和 (4.3) 表示的结果是一致的. 


\section{3 周期 Hilbert 变换}

对任意 $f(x)=\sum_{k \in \mathbb{Z}} c_{k} \mathrm{e}^{i k \frac{2 \pi}{T} x} \in L_{T}^{2}$, 式 (1.2) 中所定义的周期 Hilbert 变换可以等价地表 示为 (参见文献 $[20$, 第 1 章] 与文献 $[21$, p. 15])

$$
\tilde{H} f(x)=\sum_{k \in \mathbb{Z}}[-i(\operatorname{sgn}(k))] c_{k} \mathrm{e}^{i k \frac{2 \pi}{T} x},
$$

其中 $\operatorname{sgn}$ 表示符号函数, 即当 $x=0$ 时, $\operatorname{sgn}(x)=0$, 当 $x \neq 0$ 时, 有 $\operatorname{sgn}(x)=x /|x|$. 由于 $\dot{L}_{T}^{2} \subset \mathscr{D}_{H}^{\prime}$, 对任意 $f \in \dot{L}_{T}^{2}$, 我们将证明 $\tilde{H} f=H f$. 首先计算 $\sin (\omega x)$ 和 $\cos (\omega x)$ 的 Hilbert 变 换, 其中 $\omega \in \mathbb{R} \backslash\{0\}$.

引理 4.4 设 $\phi \in \mathscr{D}_{H}$, 则 $\hat{\phi} \in C^{\infty}(\mathbb{R} \backslash\{0\})$.

证明 对任意的 $\phi \in \mathscr{D}$, 显然有 $\hat{\phi} \in C^{\infty}(\mathbb{R} \backslash\{0\})$. 下面设 $\phi=H \psi$, 其中 $\psi \in \mathscr{D}$. 由于

$$
\hat{\phi}(\omega)=(H \psi)^{\wedge}(\omega)=-i \operatorname{sinn}(\omega) \hat{\psi}(\omega),
$$

所以 $\hat{\phi} \in C^{\infty}(\mathbb{R} \backslash\{0\})$.

定理 4.5 设 $\omega \in \mathbb{R} \backslash\{0\}$, 则 $H \mathrm{e}^{i \omega x}=-i \operatorname{sgn}(\omega) \mathrm{e}^{i \omega x}$ 对几乎处处的 $x \in \mathbb{R}$ 成立, 即

$$
H \cos (\omega x)=\operatorname{sgn}(\omega) \sin (\omega x), \quad H \sin (\omega x)=-\operatorname{sgn}(\omega) \cos (\omega x), \quad \text { a.e. } x \in \mathbb{R} .
$$

证明 对 $\omega \in \mathbb{R} \backslash\{0\}$, 有

$$
\begin{aligned}
\left\langle H \mathrm{e}^{-i \omega \cdot}, \phi\right\rangle & =-\left\langle\mathrm{e}^{-i \omega \cdot}, H \phi\right\rangle=-\lim _{A, B \rightarrow \infty} \int_{[-A, B]} \mathrm{e}^{-i \omega x} H \phi(x) d x \\
& =-(H \phi)^{\wedge}(\omega)=i \operatorname{sgn}(\omega) \hat{\phi}(\omega) \\
& =i \operatorname{sgn}(\omega)\left\langle\mathrm{e}^{-i \omega \cdot}, \phi\right\rangle \quad\left(\forall \phi \in \mathscr{D}_{H}\right),
\end{aligned}
$$

所以 $H \mathrm{e}^{-i \omega \cdot}=i(\operatorname{sgn} \omega) \mathrm{e}^{-i \omega \cdot}(\forall \omega \in \mathbb{R} \backslash\{0\})$.

定理 4.6 对任意 $f=\sum_{k \in \mathbb{Z} \backslash\{0\}} c_{k} \mathrm{e}^{i k \frac{2 \pi}{T}} \cdot \in \dot{L}_{T}^{2}$, 有

$$
H f=\sum_{k \in \mathbb{Z} \backslash\{0\}}[-i \operatorname{sgn}(k)] c_{k} \mathrm{e}^{i k \frac{2 \pi}{T} .} .
$$

证明 根据 $\dot{L}_{T}^{2} \subseteq \mathscr{D}_{H}^{\prime}$ 和定理 4.5 有

$$
H f=\sum_{k \in \mathbb{Z} \backslash\{0\}} c_{k} H \mathrm{e}^{i k \frac{2 \pi}{T}}=\sum_{k \in \mathbb{Z} \backslash\{0\}}[-i \operatorname{sgn}(k)] c_{k} \mathrm{e}^{i k \frac{2 \pi}{T}} .
$$

\section{$5 \mathscr{D}_{H}^{\prime}$ 上 Hilbert 变换的应用}

\section{1 信号解调与 Bedrosian 等式}

设 $s \in L^{2}(\mathbb{R})$ 是实值信号. 定义其瞬时振幅和瞬时相位的经典方法就是把 $s$ 表示为

$$
s(t)=\rho(t) \cos \theta(t),
$$

使得 $a(t)=\rho(t) \mathrm{e}^{i \theta(t)}$ 成为一个解析信号 $[2,22,23]$, 这里 $\rho(t) \geqslant 0$. 即

$$
H(\rho(t) \cos \theta(t))=\rho(t) \sin \theta(t),
$$

其中 $H$ 是式 (1.1) 所定义的经典意义下的 Hilbert 变换. 式 (5.1) 的左边是两个函数之乘积 的 Hilbert 变换. 正象许多作者所说 ${ }^{[23]}$, 为研究式 (5.1), 我们可以在适当的条件下应用如下 的 Bedrosian 等式:

$$
H(f g)=f H g
$$


关于 Bedrosian 等式成立的条件有若干研究 (参见 $[5,6,24,25,26]$ ), 例如对 $f, g \in L^{2}(\mathbb{R}$ ), 若 存在某个 $B>0$, 使得 $\operatorname{supp} \hat{f} \subset[-B, B], \operatorname{supp} \hat{g} \subset \mathbb{R} \backslash[-B, B]$, 那么 Bedrosian 等式成立. 在文 献 [23] 中, 作者令 $f(t)=\rho(t), g(t)=\cos \theta(t)$, 然后直接利用 Bedrosian 等式 (5.2) 推出

$$
H(\rho(t) \cos \theta(t))=\rho(t) H \cos \theta(t) .
$$

通常情形下, 由于 $\cos \theta(t)$ 不属于 $L^{2}(\mathbb{R})$, 所以 Bedrosian 等式 (5.2) 不能用来推导式 (5.3). 事实上, 因为 $s(t)=\rho(t) \cos \theta(t) \in L^{2}(\mathbb{R})$, 式 (5.3) 左边的 Hilbert 变换是按式 (1.1) 定义的, 而 式 (5.3) 的右边, 由于 $\cos \theta \notin L^{p}(\mathbb{R})$, 所以通常情形下 $H \cos \theta(t)$ 按式 (1.1) 没有意义.

若 $\theta(t)$ 满足定理 3.7 的条件, 则 $\cos \theta(t) \in \mathscr{D}_{H}^{\prime}$, 此时式 (5.3) 的两边都有意义. 设 $A$ 是解 析信号算子, 即: $A \phi=\phi+i H \phi\left(\forall \phi \in \mathscr{D}_{H}\right)$ (参见文献 $\left.[2,22]\right)$. 记

$$
\mathscr{D}_{A}^{\prime}:=\left\{f \in \mathscr{D}_{H}^{\prime} \mid\langle f, A \phi\rangle=0\left(\forall \phi \in \mathscr{D}_{H}\right)\right\} .
$$

利用定理 3.7, 我们得到下面的 Bedrosian 定理:

定理 5.1 设 $\rho \in L^{2}(\mathbb{R})$ 且 $\rho(x) \geqslant 0, \theta$ 满足定理 3.7 的条件, 则

$$
H(\rho(x) \cos \theta(x)=\rho(x) H \cos \theta(x)=\rho(x) \sin \theta(x)
$$

当且仅当 $\rho \mathrm{e}^{i \theta}, \mathrm{e}^{i \theta} \in \mathscr{D}_{A}^{\prime}$.

证明 易证 $H \cos \theta(x)=\sin \theta(x)$ 当且仅当 $H \mathrm{e}^{i \theta(x)}=-i \mathrm{e}^{i \theta(x)}$, 这等价于对任意 $\phi \in \mathscr{D}_{H}$, $-\left\langle\mathrm{e}^{i \theta}, H \phi\right\rangle=-\left\langle\mathrm{e}^{i \theta}, i \phi\right\rangle$, 即 $\mathrm{e}^{i \theta} \in \mathscr{D}_{A}^{\prime}$.

类似地, 易证 $H \cos \theta(x)=\sin \theta(x)$ 当且仅当 $\rho \mathrm{e}^{i \theta} \in \mathscr{D}_{A}^{\prime}$. 定理证毕.

作为一个特例, 我们有

推论 5.2 设 $\rho \in L^{2}(\mathbb{R})$ 且 $\rho(x) \geqslant 0, \omega>0, \theta \in \mathbb{R}$, 则

$$
H(\rho(x) \cos (\omega x+\theta)=\rho(x) H \cos (\omega x+\theta)=\rho(x) \sin (\omega x+\theta)
$$

当且仅当 $\operatorname{supp} \hat{\rho} \subset[-\omega, \omega]$.

证明 易证 $\theta(x)=\omega x+\theta$ 满足定理 3.7 的条件, 等式 $\left\langle\mathrm{e}^{i(\omega x+\theta)}, A \phi\right\rangle=\mathrm{e}^{i \theta}(A \phi)^{\wedge}(-\omega)=$ $0\left(\forall \phi \in \mathscr{D}_{H}\right)$ 蕴含 $\mathrm{e}^{i(\omega \cdot+\theta)} \in \mathscr{D}_{A}^{\prime}$. 因此式 (5.5) 成立当且仅当 $\rho \mathrm{e}^{i(\omega \cdot+\theta)} \in \mathscr{D}_{A}^{\prime}$, 也就是 $\left\langle\rho \mathrm{e}^{i \omega}, \phi+\right.$ $i H \phi\rangle=0\left(\forall \phi \in \mathscr{D}_{H}\right)$, 这等价于 $\rho(x) \mathrm{e}^{i \omega x}+i H\left[\rho(x) \mathrm{e}^{i \omega x}\right]=0$. 等式两边作 Fourier 变换得 $[\operatorname{sgn}(\xi)-1] \hat{\rho}(\xi-\omega)=0$. 利用 $\hat{\rho}$ 的 Hermite 对称性, 得 $\hat{\rho}(\xi)=0$ 对任意 $|\xi|>|\omega|$ 成立.

\section{2 算子方程}

$H: \mathscr{D}_{H}^{\prime} \rightarrow \mathscr{D}_{H}^{\prime}$ 是同胚映射的事实可以用来求解算子方程. 作为一个例子, 我们考虑以下 的方程

$$
y+H y=f,
$$

这里 $f$ 是一个已知分布. 在文献 [14] 中, Pandey 对 $f \in \mathscr{D}^{\prime}$ 研究了上面的方程的求解. 他引 入超分布空间 $H^{\prime}(\mathscr{D})$, 并把经典的 Hilbert 变换推广为算子 $H: \mathscr{D}^{\prime} \rightarrow H^{\prime}(\mathscr{D})$, 然后求得方程 (5.6) 的解 $y=\frac{1}{2}(f-H f)$. 然而这里有两个问题: 首先, Pandey 没有说明在什么空间中求解 方程 (5.6). 由于方程 (5.6) 的左边是 $y$ 与 $H y$ 之和, 这表明只有当 $y$ 和 $H y$ 在同一个空间中 时, 方程才有意义. 其次, Pandey 所求得的解 $y=\frac{1}{2}(f-H f)$ 出现了 $f$ 和 $H f$ 的求和, 这需要 所给定的 $f$ 及其 Hilbert 变换 $H f$ 属于同一个空间. 而如我们前面已述, 对 $f \in \mathscr{D}^{\prime}$, 按 Pandey 的定义有 $H f \in H^{\prime}(\mathscr{D}), H^{\prime}(\mathscr{D})$ 与 $\mathscr{D}^{\prime}$ 是两个不同的分布空间, 所以上述问题的求解是不严格 的. 由于本文所推广的 Hilbert 变换在 $\mathscr{D}_{H}^{\prime}$ 上是封闭的, 所以 $\mathscr{D}_{H}^{\prime}$ 是求解上述方程的一个合理 
的框架. 这样, 求解方程 (5.6) 的问题可以叙述为: 对已知分布 $f \in \mathscr{D}_{H}^{\prime}$, 求 $y \in \mathscr{D}_{H}^{\prime}$, 使得方程 (5.6) 成立.

将定义 4.1 所定义的 Hilbert 变换 $H$ 作用在方程 (5.6) 的两边, 有 $H y-y=H f$, 将此式 与方程 (5.6) 联立, 立即得到方程 (5.6) 的解 $y=\frac{1}{2}(f-H f)$.

致谢 作者衰心感谢审稿人对本文提出的宝贵意见.

\section{参考文献}

1 Mallat S. Wavelet Tour of Signal Processing. 2nd ed. San Diego: Academic Press, 1999

2 Cohen L. Time-Frequency Analysis. Englewood Cliffs, NJ: Prentice-Hall, 1995

3 Huang N E, Shen Z, Long S R, et al. The empirical mode decomposition and the Hilbert spectrum for nonlinear and non-stationary time series analysis. Proc $R$ Soc Lond Ser A, 454: 903-995 (1998)

4 Chen Q, Huang N, Riemenschneider S, et al. A B-spline approach for empirical mode decompositions. $A d v$ Comput Math, 24: 171-195 (2006)

5 Tan L, Yang L, Huang D. Necessary and Sufficient Conditions for the Bedrosian Identity. J Integral Equations Appl, in press

$6 \mathrm{Xu}$ Y, Yan D. The Bedrosian identity for the Hilbert transform of product functions. Proc Amer Math Soc, 134(9): 2719-2728 (2006)

7 Qian T. Characterization of boundary values of functions in hardy spaces with application in signal analysis. J Integral Equations Appl, 17(2): 159-198 (2005)

8 Gasquet C, Witomski P. Fourier Analysis and Applications: Filtering, Numerical Computation, Wavelets (Translated by Ryan R). New York: Springer-Verlag, 1998

9 Pinsky M A. Introduction to Fourier Analysis and Wavelets. Pacific Grove, CA: Brooks/Cole, 2002

10 Stein E M, Weiss G. Introduction to Fourier Analysis on Euclidean Spaces. Princeton: Princeton University Press, 1971

11 Beltrami E J, Wohlers M R. Distributions and the Boundary Values of Analytic Functions. New YorkLondon: Academic Press, 1966

12 Bremermann H J. Some remarks on anaytic representations and products of distributions. SIAM J Appl Math, 15(4): 920-943 (1967)

13 Orton M. Hilbert transforms, Plemelj relations, and Fourier transforms of distribution. SIAM J Math Anal, 4(4): 656-670 (1973)

14 Pandey J N. The Hilbert transform of Schwartz distributions. Proc Amer Math Soc, 89(1): 86-90 (1983)

15 Pandey J N, Chaudhry M A. The Hilbert transform of generalized functions and applications. Canad J Math, 35(3): 478-495 (1983)

16 Pandey J N, Chaudhry M A. The Hilbert transform of Schwartz distribtuions II. Math Proc Cambridge Philos Soc, 102: 553-559 (1987)

17 Rudin W. Real and Complex Analysis. 2nd ed. New Delhi: Tata McGraw-Hill, 1987

18 Folland G B. Real Analysis. New York: John Wiley \& Sons, Inc., 1984

19 Titchmarsh E C. Introduction to the Theory of Fourier Integrals. 3nd ed. New York: Chelsea Publishing Company, 1986

20 Meyer Y. Ondelettes ét Opératewrs, Vol. I. Paris: Hermann, 1990

21 Bergh J, Löfstrom J. Interpolation Spaces. Berlin-Heidelberg-New York: Springer-Verlag, 1976

22 Vakman D. On the analytic signal, the teager-kaiser energy algorithm, and other methods for defining amplitude and frequency. IEEE Trans Signal Process, 44(4): 791-797 (1996)

23 Picinbono B. On instantaneous amplitude and phase of signals. IEEE Trans Signal Process, 45(3): 552-560 (1997)

24 Bedrosian E. A product theorem for Hilbert transform. Proc IEEE, 51: 868-869 (1963)

25 Brown J L. Analytic signals and product theorems for Hilbert transforms. IEEE Trans Circuits Syst, 21(6): 790-792 (1974)

26 Brown J L. A Hilbert transform product theorem. Proc IEEE, 74: 520-521 (1986) 